\title{
Dynamique de croissance et taux de mortalité de Rhizophora spp. dans les mangroves de l'estuaire du Rio del Rey: Site de Bamusso (Sud-Ouest Cameroun)
}

\author{
NDEMA NSOMBO Eugene ${ }^{1,2^{*}}$, SONE ESSOH Willy ${ }^{1}$, GORDON Ajonina ${ }^{1,4}$, ETAME Jacques ${ }^{2}$, \\ NDONGO DIN ${ }^{3}$, DIYOUKE MIBOG Eugene 4
}

(1) Département de Gestion des Écosystèmes Aquatiques, Institut des Sciences Halieutiques (ISH), Université de Douala, B.P. 2701 Douala-Cameroun

(2) Département de Géosciences et Environnement, Faculté des Sciences, Université de Douala, B.P. 24157 Douala-Cameroun

(3) Département de Biologie des Organismes Végétaux, Faculté de Sciences, Université de Douala, B.P. 24157

Douala-Cameroun

(4) Cameroon Wildlife Conservation Society, B.P. 54 Mouanko, Region du Littoral-Cameroun

$\left.{ }^{*}\right)$ Corresponding author : ndema_eugene2002@yahoo.fr ; Tel : +237 99421742

Original submitted in on $21^{\text {st }}$ October 2014. Published online at www.m.elewa.org on $30^{\text {th }}$ January 2015 http://dx.doi.org/10.4314/jab.v85i1.7

\section{RÉSUMÉ}

Objectifs : L'objectif des présents travaux est de clarifier le stock du peuplement de Rhizophora ssp. du site par la détermination de ses paramètres de structure spatiale et d'évaluer sa croissance annuelle.

Méthodologie et résultats: La méthode d'étude est celle des transects. Deux transects de $100 \mathrm{~m} \times 10 \mathrm{~m}$ distantes de $5 \mathrm{~km}$ orientés WNW-ESE et perpendiculaires au principal chenal ont été établis au hasard .Chaque transect était subdivisé en trois placettes de $20 \mathrm{~m} \times 10 \mathrm{~m}$ et chaque placettes scindé en deux sous placettes de $20 \mathrm{~m} \times 5 \mathrm{~m}$. Les mesures (Diamètre et hauteur) ont été effectuées à l'intérieur de chaque sous placette pendant trois campagnes $(2009,2012,2014)$. Les résultats montrent que les diamètres moyens pour le transect1(T1) sont de $4,76 \pm 0,23 \mathrm{~cm} ; 5,09 \pm 0,25 \mathrm{~cm}$ et $5,43 \pm 0,28 \mathrm{~cm}$ contre $26,53 \pm 1,72 \mathrm{~cm} ; 24,66$ $\pm 1,90 \mathrm{~cm}$ et $25,26 \pm 1,93 \mathrm{~cm}$ pour le transect2 (T2) respectivement pour 2009; 2012 et 2014 . Les hauteurs moyennes varient de 4,51 $\pm 0,16 \mathrm{~m}$ (2009); 4,74 $\pm 0,18 \mathrm{~m}$ (2012) et 4,98 $\pm 0,2 \mathrm{~m}$ (2014) pour T1 contre $20,20 \pm 1,22 \mathrm{~m} ; 18,86 \pm 1,35 \mathrm{~m}$ et 19,29 $\pm 1,39 \mathrm{~m}$ pour T2. L'analyse de la croissance du peuplement montre que les diamètres d'exploitation du bois de la mangrove affectionnée par les riverains se situent dans l'intervalle $(5-30 \mathrm{~cm})$. Le test d'ANOVA montre une différence significative $(P<0,05)$ pour les paramètres, de structure et une différence non significative $(P>0,05)$ de la croissance annuelle entre $T 1$ et $T 2$.

Conclusion et application des résultats : Cette étude a montré un taux de mortalité relativement faible $(m=2,27 \%)$ qui présage d'une pression anthropique faible sur cet écosystème. L'étude de la dynamique réalisée permet de recommander ce site dans le processus REDD+ pour le calcul du carbone bleu indispensable dans la lutte contre le phénomène de changement climatique.

Mots clés: Transect, Diamètre; Hauteur; Nombre d'individus, Bamusso 


\title{
Ndema et al.. J. Appl. Biosci. Dynamique de croissance et taux de mortalité de Rhizophora spp. dans les
} mangroves de l'estuaire du Rio del Rey : Site de Bamusso (Sud-Ouest Cameroun)

\section{Growth dynamic and death rate of Rhizophora spp. within mangrove's forest of Rio del Rey estuary} (South-West Cameroon)

\begin{abstract}
Objective: This study was conducted to clarify the stock of Rhizophora ssp and to assess its growth within the study area.

Methodology and results: Transect method was carried out during this study. So, two transects of $100 \mathrm{~m}$ in length, $10 \mathrm{~m}$ in width and 0.1 ha in area each distance to $5 \mathrm{~km}$ oriented WNW-ESE and perpendicular to the main channel were established randomly . Each transect was divided in three plots of $20 \mathrm{~m} \times 10 \mathrm{~m}$ each and each plot separated in two subplots of $20 \mathrm{~m} \times 5 \mathrm{~m}$ each. Measures (Diameter and height) were collected within each subplot during three data collections campaigns $(2009,2012$,and 2014). The results show that mean diameters of transect1(T1) were $4.76 \pm 0.23 \mathrm{~cm} ; 5.09 \pm 0.25 \mathrm{~cm}$ and $5.43 \pm 0.28 \mathrm{~cm}$ to $26.53 \pm 1.72 \mathrm{~cm}$; $24.66 \pm 1.90 \mathrm{~cm}$ and $25.26 \pm 1.93 \mathrm{~cm}$ for transect2 (T2) respectively in 2009; 2012 et 2014 . Mean height values were $4.51 \pm 0.16 \mathrm{~m}(2009) ; 4.74 \pm 0.18 \mathrm{~m}(2012)$ and $4.98 \pm 0.2 \mathrm{~m}$ (2014) for T1 to $20.20 \pm 1.22 \mathrm{~m}$; $18.86 \pm 1.35 \mathrm{~m}$ and $19.29 \pm 1.39 \mathrm{~m}$ for T2. Study of growth show that used diameters of mangrove woods by neighborhood are inside class $5-30 \mathrm{~cm}$. The one-way ANOVA test showed a significant difference $(P<0.05)$ for structure parameters and no significant difference $(P>0,05)$ for annual growth between $T 1$ and T2.

Conclusion and application of findings: This study showed a relatively weak annual death rate of trees $(m=2.27 \%)$ dues to a weak anthropogenic pressure in this ecosystem. This dynamic study will recommend the site to the REDD+ process in order to estimate its blue carbon stock important to fight against climate change.
\end{abstract}

Key words: Transect, Diameter, Height, Number of individuals, Bamusso

\section{INTRODUCTION}

Les mangroves se rencontrent le long des côtes tropicales et subtropicales où l'on trouve de l'eau saumâtre à salinité variable et une faune diversifiée (Tomlinson, 1986 ; Duke, 1992 ; Jayatissa et al., 2002 ; Cannicci et al., 2008; Nagelkerken et al., 2008). Longtemps considérées comme des milieux inhospitaliers (Thevand, 2002), les mangroves ont commencé depuis quelques décennies à être reconnues " d'utilité publique ». En effet, elles présentent un intérêt tant sur le plan économique (Kovacs, 1999) qu'écologique. Écotone entre milieu terrestre et marin, l'écosystème de mangrove constitue une zone d'une grande originalité structurale et fonctionnelle (Betoulle, 1998), jouant un rôle dans les chaînes trophiques côtières (Dittmar et al., 2001; Laedgaard \& Johnson, 2001 ) et constituant un important puits de carbone (Twilley et al., 1992; Gattuso et al., 1998). Les travaux les plus récentes sur les mangroves prouvent la capacité de cet écosystème à juguler le phénomène de changement climatique par la séquestration $d u$ carbone de ces différents composantes (Trevor et al, 2014; Adame et al, 2013; Donato et al, 2012; Lovelock et al, 2011). En outre, les mangroves jouent un rôle de barrière contre les catastrophes naturelles. C'est ainsi qu'elles protègent les populations environnantes des dommages que peuvent causés les cyclones, les tsunamis et les ouragans (Dahdouh-Guebas, 2006 ; Alongi, 2008). Elles produisent également des biens et services ainsi qu'un revenu à la société humaine (Krauss et al., 2008). En limitant l'érosion côtière, cet écosystème participe à l'avancée de la terre vers l'océan tout en constituant une zone tampon dans les régions sujettes aux tempêtes et aux cyclones (Hong et al, 1993). Les mangroves constituent aussi une importante source de revenues et plusieurs autres activités de subsistance et commerciales telles que la pêche et l'exploitation de bois y sont exercées (Din et al., 2006). A cause de ces nombreux biens et services, l'utilité de préserver la biodiversité des mangroves par le suivi de ses paramètres de croissance apparaît 


\section{Ndema et al.. J. Appl. Biosci. Dynamique de croissance et taux de mortalité de Rhizophora spp. dans les}

mangroves de l'estuaire du Rio del Rey : Site de Bamusso (Sud-Ouest Cameroun)

clairement. Plusieurs études montrent qu'entre 5 $\%$ et $85 \%$ environ de la superficie originale des mangroves a été perdue particulièrement pendant la seconde moitié du 20 e siècle. Les perspectives d'un monde sans mangrove s'apparentent être une réalité (Duke et al., 2007) car les pressions anthropiques le long des côtes sont en pleine croissances (Walters et al., 2008). Les mangroves de l'Afrique subissent d'énormes pressions au cours des dernières décennies, au point qu'en Afrique occidentale et centrale, 20 à $30 \%$ des mangroves ont disparu en 25 ans. Cela est dû à plusieurs facteurs, en particulier à l'urbanisation par le développement des infrastructures et les résidences, à l'exploitation des carrières de sel et de sable, à la pollution provoquée par les industries, les produits agrochimiques industriels et l'exploitation du pétrole et du gaz, à l'absence d'une législation appropriée, au déboisement pour le fumage de poisson [Ajonina et Usongo, 2001 ; Ajonina et al., 2005], à la prolifération d'espèces invasives et aux effets du changement climatique, accentués par la croissance démographique. Le long des côtes camerounaises, les mangroves couvrent une superficie actuellement estimée à

\section{MATÉRIEL ET METHODES}

Site d'étude : L'étude a été menée dans la foret de mangrove de la localité de Bamusso (Estuaire du Rio del Rey). Chef lieu d'arrondissement, Bamusso est une presque île située dans le département du Ndian, région du Sud-ouest. Limité au Sud et l'Ouest par I'océan Atlantique, au nord par Ekondo titi et à l'Est par le département de la Meme et celui du Fako (Fig. 1), cette localité de coordonnées géographiques $4^{\circ} 45^{\prime}$ $4^{\circ} 50^{\prime}$ de latitude Nord et $8^{\circ} 30^{\prime}-9^{\circ} 00^{\prime}$ de longitude Est présente une végétation de forêt sempervirente dominée par la mangrove et entrecoupé par une prairie arbustive ( Ajonina, 2011). Cette forêt de mangrove dominée par la famille des Rhizophoracées est disséquée par un grand nombre de chenaux qui constituent des voies de circulation pour les pêcheurs, les braconniers et touristes. Le climat de la région est influencé par la proximité de l'océan atlantique et du Mont Cameroun d'une part, et d'autre part, par l'équateur météorologique où convergent l'anticyclone des Açores depuis l'hémisphère Nord et celui de Sainte Hélène venant du sud (Din, 2001). Ce climat appartient environ 1961,84 km² (Spalding et al., 2010). Elles sont distribuées principalement entre deux estuaires (estuaire du Rio del Rey et estuaire du Cameroun) mais sont présentes également sous forme de petits peuplements à superficies variables dans la région du Sud-Cameroun (Estuaire du Rio Ntem). Si les mangroves de l'Estuaire du Cameroun ont été étudiées par de nombreux auteurs, celles de l'Estuaire du Rio del Rey restent très peu connues à cause du conflit frontalier de Bakassi qui opposait le Cameroun au Nigéria. La zone est restée longtemps un domaine militaire interdit d'accès pour les civils et notamment pour les chercheurs. Cette zone très productive est riche en pétrole et en poissons et doit faire l'objet de véritables recherches scientifiques sur le terrain. Fort de ce qui précède l'objectif de la présente étude est d'évaluer le stock du peuplement de Rhizophora ssp du site par la détermination de la structure de la végétation. En outre la dynamique de la population sera étudiée à travers le calcul du taux de croissance et de mortalité annuelle pendant les différentes campagnes.

au domaine équatorial de type littoral ou "Camerounien" qui se caractérise par deux saisons avec une longue saison de pluies (mars à novembre) qui peut totalement effacer la saison sèche toujours ponctuée de pluies. Cette saison sèche théorique(novembre à février) apparaît dans plusieurs localités comme un simple ralentissement de pluies. Bamusso présente des précipitations moyennes annuelles de l'ordre de $3800 \mathrm{~mm}$. Elles varient mensuellement de $90 \mathrm{~mm}$ pendant la saison sèche et $330 \mathrm{~mm}$ en saison des pluies et le nombre annuel de jours de pluies est toujours supérieur à 250 partout dans l'estuaire du Rio del Rey. En outre, l'évaporation moyenne mensuelle est de $120 \mathrm{~mm}$. La salinité dont plusieurs espèces de mangrove doivent gérer la variation est modérée et est toujours inférieure à $35 \%$ avec une moyenne qui attend rarement $20 \%$ dans les estuaires. Les cours d'eau les plus importants sur le site sont la moko, le Ndian et la mémé.

La composante sociologique est formée des allogènes et des autochtones. Ces derniers sont originaires de Pitti dans la Sanaga maritime région du Littoral alors 
Ndema et al.. J. Appl. Biosci. Dynamique de croissance et taux de mortalité de Rhizophora spp. dans les mangroves de l'estuaire du Rio del Rey : Site de Bamusso (Sud-Ouest Cameroun)

que les allogènes sont constitués des Mosgoum venus de la partie Nord du Cameroun pour rivaliser la forte communauté Nigériane dans la pratique de la principale activité source de revenu qu'est la pêche. Alors que les autochtones considèrent les mangroves comme des sites sacrés consacrés aux divers rites, les allogènes les utilisent pour la construction, le fumage du poisson et dans une moindre mesure pour l'agriculture. 


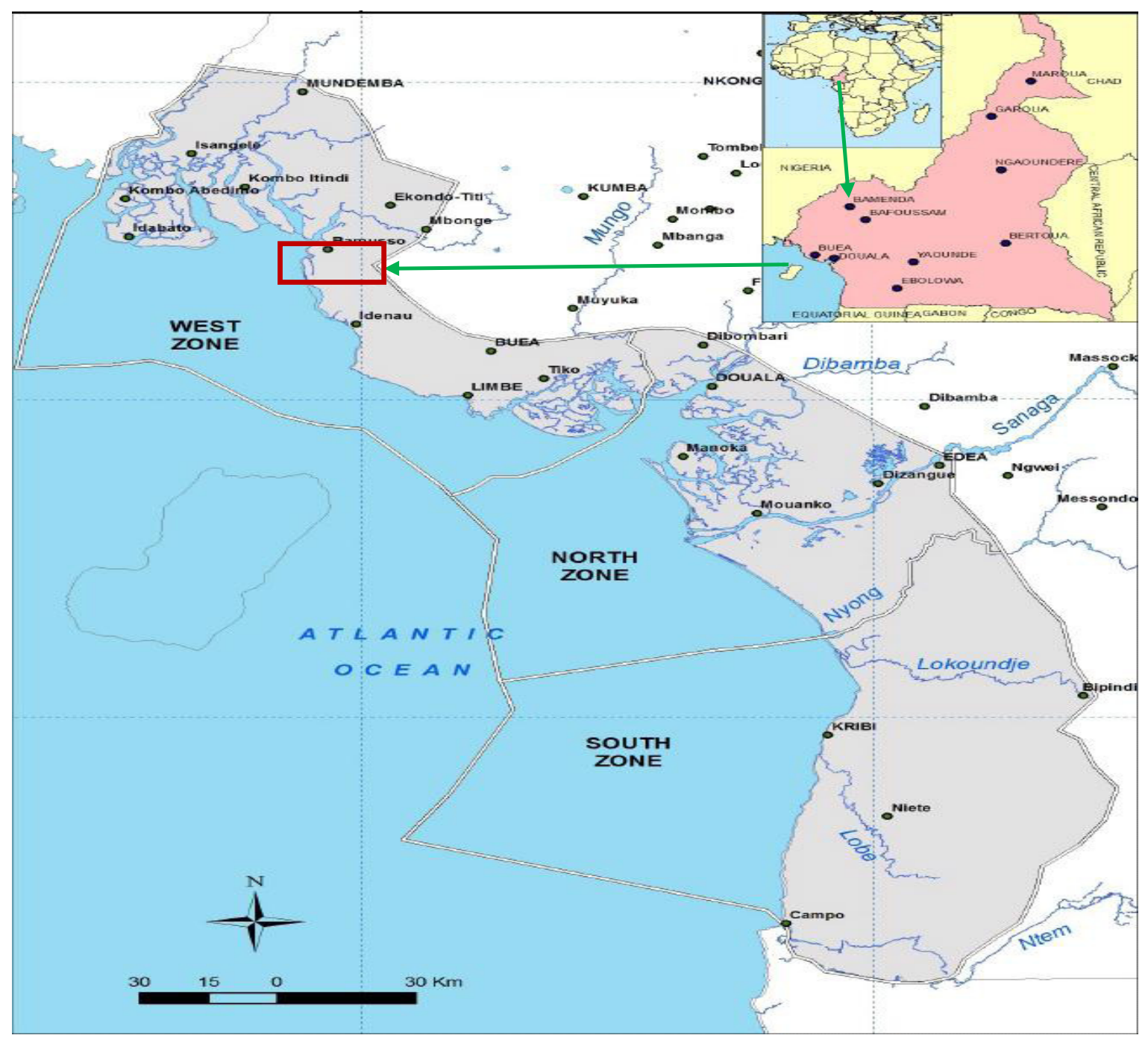

LEGENDE:<smiles>C1CCCCC1</smiles>

\section{Bamusso}

Limites des zones de mangrove

Océan atlantique

Figure 1: Localisation du site d'étude (modifiée de Ajonina 2011)

Échantillonnage : La méthode d'échantillonnage est celle des transects (Imbert, 1985). Deux transects de $100 \mathrm{~m}$ de long sur $10 \mathrm{~m}$ de large soit une superficie de $1000 \mathrm{~m}^{2}$ chacun ont été établis au hasard. Ils étaient distante de $5 \mathrm{~km}$ avec des lignes de base orientées WSW-ENE et perpendiculaires au principal chenal. Chaque transect a été subdivisé en trois placettes de $20 \mathrm{~m} \times 10 \mathrm{~m}$ avec un écart de $10 \mathrm{~m}$ et Chaque placette à son tour a été scindé en deux sous placettes de $20 \mathrm{~m} \times 5 \mathrm{~m}$ (Fig.2). Les mesures de diamètre et hauteur se sont effectués pendant trois campagnes ( 2009 ,
2012 et 2014) à l'intérieur des douze sous placettes. Tous les individus de Rhizophora spp. à partir d'un centimètre de diamètre ont été répertoriés et mesurés. Lorsque l'arbre le plus proche était repéré, la mesure du DBH (Diameter at Breast Height) était prise à $D_{130}$ $(1,30 \mathrm{~m}$ du sol) par un pied à coulisse si le diamètre ne dépassait pas $10 \mathrm{~cm}$ ou par un ruban forestier pour les individus de diamètre supérieur à $10 \mathrm{~cm}$. Dans le cas contraire, le diamètre était mesuré à $30 \mathrm{~cm}$ au dessus des racines échasses après avoir grimpé sur l'arbre (Fig.3). 


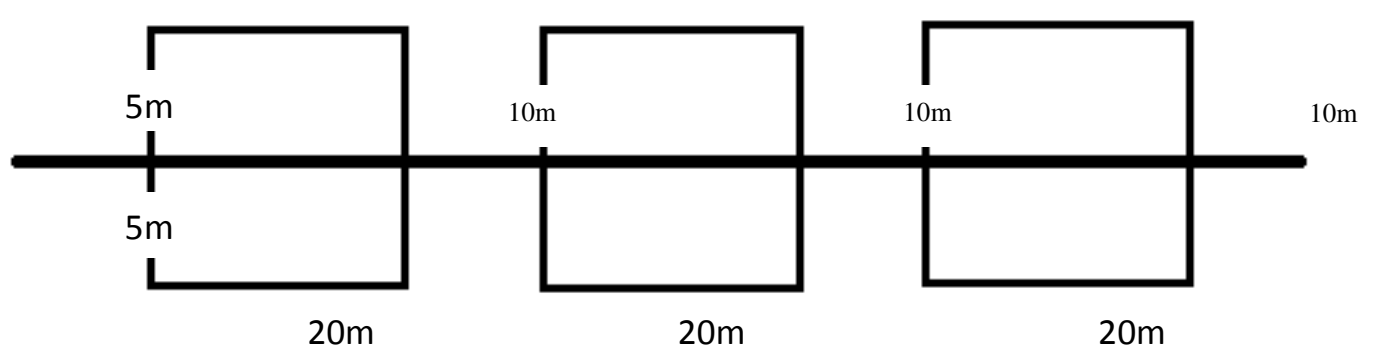

Ligne de base

Figure 2: Positionnement des placettes dans le transect
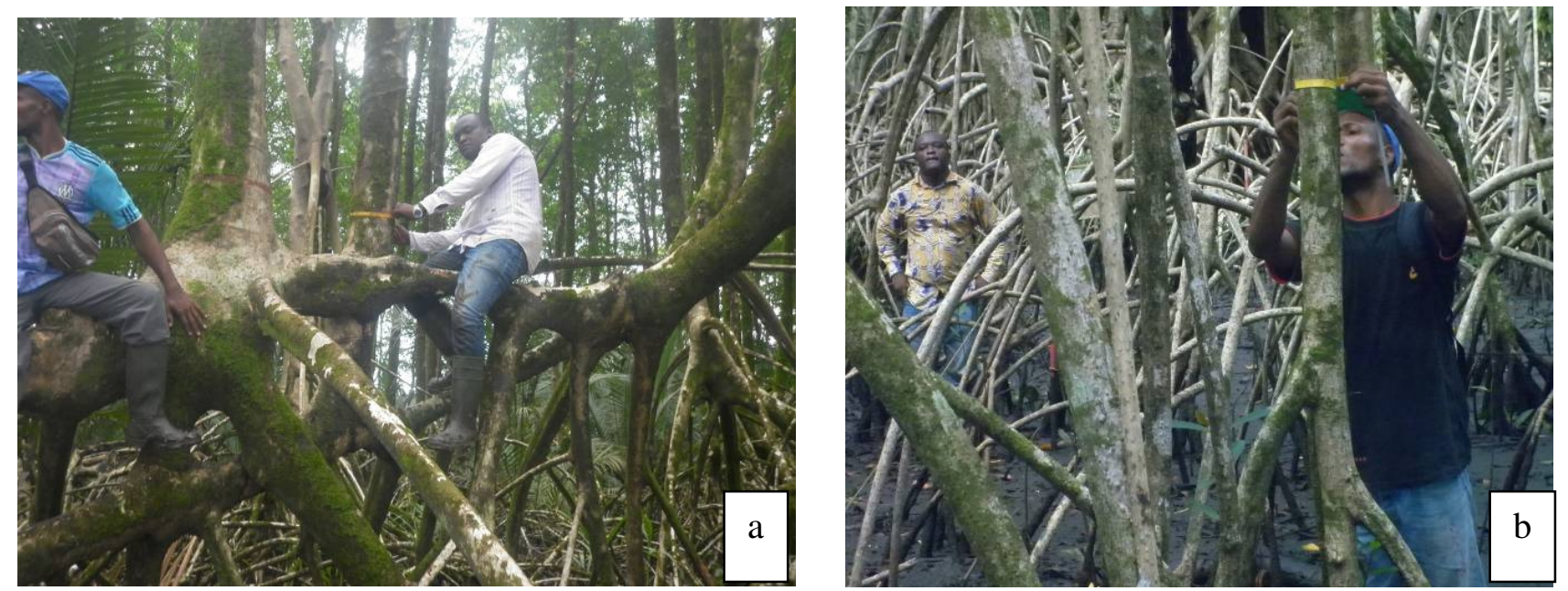

Figure 3: Mesure des diamètres: a-Après grimpade ; b-Au sol

La hauteur de certains arbres a été estimé à l'aide du clinomètre. Cette estimation, complétée par la mesure des longueurs des arbres tombés a permis d'établir une relation entre le diamètre et la hauteur. L'équation établie a permis d'extrapoler les valeurs des hauteurs manquantes pour Rhizophora spp. Pour éviter de compter ou mesurer un arbre deux fois, chaque

la densité exprimée en individus par hectare : $D a=$ individu inventorié était marqué à la peinture rouge et étiqueté. Les paramètres qui n'ont pas été mesurés ont été obtenus par calcul à partir des mesures effectuées sur le terrain. Ainsi, la densité absolue, la surface basale, le volume du peuplement, la croissance annuelle par période et le taux de mortalité ont été obtenus à partir des équations suivantes :

$$
{ }_{S p}^{N} \times 10000
$$

avec $D a$ (densité absolue), $N$ (nombre d'individus de la parcelle), $S p$ (Surface de la parcelle en $\mathrm{m}^{2}$ ); la surface basale ou surface terrière exprimée en mètre carrée par hectare

$$
\begin{array}{ll} 
& S b=\left(\frac{D_{\text {moy }}}{2}\right)^{2} \times 3,14 \times 10000 ; D_{\text {moy }} \text { étant le diamètre moyen de la zone; } \\
\text { - } & \text { le volume du peuplement }(V p) \text { en } \mathrm{m}^{3} / \mathrm{ha} \\
& V p=S b \times \mathrm{H} \times 0,6 ; \text { avec } \mathrm{H} \text { la hauteur des arbres et } 0,6 \text { le facteur de forme } \\
\text { - } & \text { la croissance annuelle par période des diamètre }(\mathrm{Cad}) \text { et des hauteurs (Cah) exprimée en } \mathrm{cm} / \mathrm{an}
\end{array}
$$




$$
C a d=\quad \frac{D-D o}{t-t o} \quad C h=\quad \frac{H-H o}{t-t o} D \text { et } H \text { étant les diamètres et hauteurs à l'instant }
$$

$t$; Do et $H o$ les diamètres et hauteurs initiaux (temps to)

- $\quad$ la croissance annuelle par période de la surface basale (Cas) et du volume (Cav)

$$
\text { Cas }=\quad \frac{S-S O}{t \text {-to }}: \text { Cav }=\quad \frac{V \text {-Vo }}{t \text { - } t o}
$$

Cas en $\mathrm{m}^{2} / \mathrm{ha} / \mathrm{an}$ et Cav en $\mathrm{m}^{3} / \mathrm{ha} / \mathrm{an}$; $S$ et $V$ étant les surfaces terrières et volumes à l'instant $t$; So et Vo les surfaces et volumes initiaux (temps to)

- $\quad$ le taux de mortalité $m$ exprimé en pourcentage par unité de temps est défini par l'équation différentielle :

$$
\begin{aligned}
& \frac{d N}{d t}=-m N \text { ou } \frac{d N}{N}=-m d t \\
& \frac{\left(N-N_{0}\right)}{N\left(t-t_{0}\right)}=\frac{\Delta N}{N \Delta t}=-m
\end{aligned}
$$

$N$ étant le nombre d'individus à l'instant $t$ et No le nombre d'individu initial (temps to ).

Analyse des données collectées: Les données collectées ont été traitées et analysées statistiquement par deux approches : l'analyse statistique descriptive et l'inférence statistique. L'analyse statistique descriptive a consisté à ranger les données dans le tableur EXCEL, et à obtenir les résultats sous forme de

\section{RÉSULTATS}

\section{Structure de la végétation}

Stock du peuplement: En 2009, année de la première campagne, le transect1 (T1) a présenté un diamètre moyen de $4,76 \pm 0,23 \mathrm{~cm}$ avec une valeur minimale (Dmin) de l'ordre de $1,07 \mathrm{~cm}$ pour une valeur maximale (Dmax) de $14,3 \mathrm{~cm}$. Le diamètre moyen du transect 2 (T2) quant à lui était de $26,53 \pm 1,72 \mathrm{~cm}$ avec un Dmin de $6,6 \mathrm{~cm}$ et un Dmax de $75,1 \mathrm{~cm}$. Les moyennes des hauteurs étaient respectivement de $4,5 \pm 0,16 \mathrm{~m}$ et $20,20 \pm 1,22 \mathrm{~m}$ pour T1 et T2. Le minimum des hauteurs ( $\mathrm{Hmin}$ ) de valeur $1,83 \mathrm{~m}$ était obtenu sur le transect 1 alors que la hauteur maximale (Hmax) de $55,24 \mathrm{~m}$ était attribué au transect 2 . Les diamètres moyens en 2012 et 2014 sont respectivement de tableaux et graphiques. L'analyse statistique inférentielle s'est faite à l'aide du programme SPSS ; ce dernier a servi à déterminer s'il existe une différence significative entre les paramètres de structure des différents transects.

$5,09 \pm 0,25 \mathrm{~cm}$ (T1) et $24,66 \pm 1,90 \mathrm{~cm}(\mathrm{~T} 2)$ puis $5,43 \pm 0,28 \mathrm{~cm}$ (T1) et $25,26 \pm 1,93 \mathrm{~cm}$ (T2). En 2012 et 2014, les hauteurs moyennes sont respectivement de $4,74 \pm 0,18 \mathrm{~m}$ et $4,98 \pm 0,2 \mathrm{~m}$ pour le transect 1 contre $18,86 \pm 1,35 \mathrm{~m}$ et $19,29 \pm 1,39 \mathrm{~m}$ pour le transect 2 . Le nombre d'individus ( $\mathrm{N}$ ) total répertoriés en 2009 dans les deux transects est de 179 soit 158 pour le transect 1 et 21 pour le transect 2. La densité absolue était de $2633 \mathrm{~N} /$ ha pour le transect 1 et $350 \mathrm{~N} / \mathrm{ha}$ pour le transect 2. En 2012 cet densité est de $2500 \mathrm{~N} / \mathrm{ha}$ et $333 \mathrm{~N} /$ ha respectivement pour $\mathrm{T} 1$ et $\mathrm{T} 2$ contre 2333 $\mathrm{N} / \mathrm{ha}$ (T1) et $333 \mathrm{~N} / \mathrm{ha}$ (T2) en 2014 (Tableau 1). 
Ndema et al.. J. Appl. Biosci. Dynamique de croissance et taux de mortalité de Rhizophora spp. dans les mangroves de l'estuaire du Rio del Rey : Site de Bamusso (Sud-Ouest Cameroun)

Tableau 1: Répartition des paramètres de structure du site d'étude

\begin{tabular}{|c|c|c|c|c|c|c|c|c|c|c|c|c|c|}
\hline \multirow{3}{*}{ Année } & \multirow{3}{*}{ Transect } & \multicolumn{12}{|c|}{ Paramètres } \\
\hline & & \multicolumn{4}{|c|}{ Diamètres $(\mathrm{cm})$} & \multicolumn{4}{|c|}{ Hauteurs (m) } & \multirow{2}{*}{$\begin{array}{l}\text { Nombre } \\
\text { d'individus } \\
\text { (N) }\end{array}$} & \multirow{2}{*}{$\begin{array}{l}\text { Densité } \\
\text { Absolue } \\
\text { (N/ha) }\end{array}$} & \multirow{2}{*}{$\begin{array}{l}\text { Surface } \\
\text { basale } \\
\left(\mathrm{m}^{2} / \mathrm{ha}\right)\end{array}$} & \multirow{2}{*}{$\begin{array}{l}\text { Volume } \\
\left(\mathrm{m}^{3} / \mathrm{ha}\right)\end{array}$} \\
\hline & & Dmin & Dmax & Dmoy & EC & Hmin & Hmax & Hmoy & EC & & & & \\
\hline \multirow{3}{*}{2009} & T1 & 1,07 & 14,3 & 4,76 & 0,23 & 1,83 & 11,38 & 4,5 & 0,16 & 158 & 2633 & 0,38 & 1,56 \\
\hline & $\begin{array}{l}\text { T2 } \\
\text { Total }\end{array}$ & 6,6 & 75,1 & 26,53 & 1,72 & 5,82 & 55,24 & 20,20 & 1,22 & $\begin{array}{l}21 \\
179\end{array}$ & $\begin{array}{l}350 \\
2983\end{array}$ & $\begin{array}{l}1,48 \\
1,86\end{array}$ & $\begin{array}{l}28,89 \\
30,45\end{array}$ \\
\hline & $\mathrm{T} 1$ & 1,3 & 14,3 & 5,09 & 0,25 & 2 & 11,38 & 4,74 & 0,18 & 150 & 2500 & 0,40 & 1,69 \\
\hline \multirow{2}{*}{2012} & T2 & 7,6 & 43,2 & 24,66 & 1,90 & 6,55 & 32,23 & 18,86 & 1,35 & 20 & 333 & $\begin{array}{l}1,08 \\
148\end{array}$ & 15 \\
\hline & T1 & 1,3 & 14,9 & 5,43 & 0,28 & 2 & 11,81 & 4,98 & 0,2 & $\begin{array}{l}1 / 0 \\
140\end{array}$ & $\begin{array}{l}2000 \\
2333\end{array}$ & $\begin{array}{l}1,40 \\
0,42\end{array}$ & 1,84 \\
\hline 2014 & $\begin{array}{l}\text { T2 } \\
\text { Total }\end{array}$ & 8,8 & 43,9 & 25,26 & 1,93 & 7,41 & 32,73 & 19,29 & 1,39 & $\begin{array}{l}20 \\
160\end{array}$ & $\begin{array}{l}333 \\
2666\end{array}$ & $\begin{array}{l}1,12 \\
1,54\end{array}$ & $\begin{array}{l}15,92 \\
17,76\end{array}$ \\
\hline
\end{tabular}



mangroves de l'estuaire du Rio del Rey : Site de Bamusso (Sud-Ouest Cameroun)

La densité a eu une évolution décroissante avec le temps dans le transect 1 alors qu'elle est resté quasi stable dans le transect 2. L'évolution décroissante avec le temps de la densité absolue dans le transect1 peut s'expliquer par la mortalité de certains arbres due à la compétition intraspécifique par rapport à la lumiere et aux substances nutrives. La quasi stabilité de la densité du transect2 traduit sa maturité ce qui laisse croire que cette foret aurait atteint le stade climacique où la compétition est quasi nulle. La surface basale moyenne était de $0,38 \mathrm{~m}^{2} /$ ha pour T1 et 1,48 $\mathrm{m}^{2} /$ ha pour T2 en 2009. Elle est de l'ordre de $0,4 \mathrm{~m}^{2} / \mathrm{ha}$ (T1) et $1,08 \mathrm{~m}^{2} / \mathrm{ha}$ (T2) en 2012 contre $0,42 \mathrm{~m}^{2}$ (T1) et 1,12 $\mathrm{m}^{2} / \mathrm{ha}$ (T2) en 2014. Ces valeurs montrent une évolution croissante de la surface basale avec le temps pour T1 alors que cette évolution est décroissante entre 2009 et 2012 puis croissante entre 2012 et 2014 dans T2. La variation décroissante dans T2 de 2009 à 2012 se justifierait par la disparition d'un arbre de grand diamètre $(\mathrm{d}=75,1 \mathrm{~cm})$ à cette période (Tableau 1).

Le volume du peuplement était de $1,56 \mathrm{~m}^{3} / \mathrm{ha}$ (T1) et $28,89 \mathrm{~m}^{3} / \mathrm{ha}$ (T2) en 2009. En 2012, il est de 1,69 (T1) et $15 \mathrm{~m}^{3} / \mathrm{ha}$ (T2) contre 1,84 (T1) et $15,92 \mathrm{~m}^{3} / \mathrm{ha}$ en 2014. L'application du test d'anova montre une différence très significative $(P<0,01)$ entre les valeurs des diamètres moyens des transect1 et 2. Les surfaces basales entre ces deux transects montrent aussi une différence significative $(P=0,011)$ alors que les volumes du peuplement ont des différences non significatives $(p=0,056)$.

Distribution des classes de Diamètre et hauteur : La distribution du nombre d'individus $(\mathrm{N})$ par classe de diamètre montre lors de la première campagne (2009) que les individus se répartissaient en trois classes: classe I: $1 \leq \mathrm{D}<5 \mathrm{~cm}$; classe II: $5 \leq \mathrm{D}<10 \mathrm{~cm}$; classe III: $10 \leq \mathrm{D}<30 \mathrm{~cm}$ pour le transect1 (T1) et en quatre classes pour le transect2 (T2): classe II:5 $\leq \mathrm{D}<10 \mathrm{~cm}$; classe III : $10 \leq D<30 \mathrm{~cm}$; classe IV: $30 \leq D<50$ et classe $\mathrm{V}$ : $50 \leq \mathrm{D}<100$. La classe I $(1 \leq \mathrm{D}<5 \mathrm{~cm})$ n'est pas représentée dans T2 alors qu'elle constitue la classe de diamètre modale pour $\mathrm{T} 1(\mathrm{~N}=98)$. Le pourcentage d'individus compris dans cette classe de diamètre par rapport au individus du transect1 ( $\mathrm{N}=158)$ est de $62,02 \%$ et de $54,75 \%$ par rapport au nombre total d'individus du site $(\mathrm{N}=179)$. La classe de diamètre la plus grande pour le transect 1 est $10-30 \mathrm{~cm}(\mathrm{~N}=10$; $6,30 \%$ du nombre d'individus total du transect) contre $50-100 \mathrm{~cm}(\mathrm{~N}=1 ; 4,76 \%)$ pour le transect2 $(\mathrm{N}=21)$ qui a pour classe modale $10-30 \mathrm{~cm} \quad(\mathrm{~N}=14 ; 66,66 \%$ du nombre total d'individus du transect) (Fig. 4).

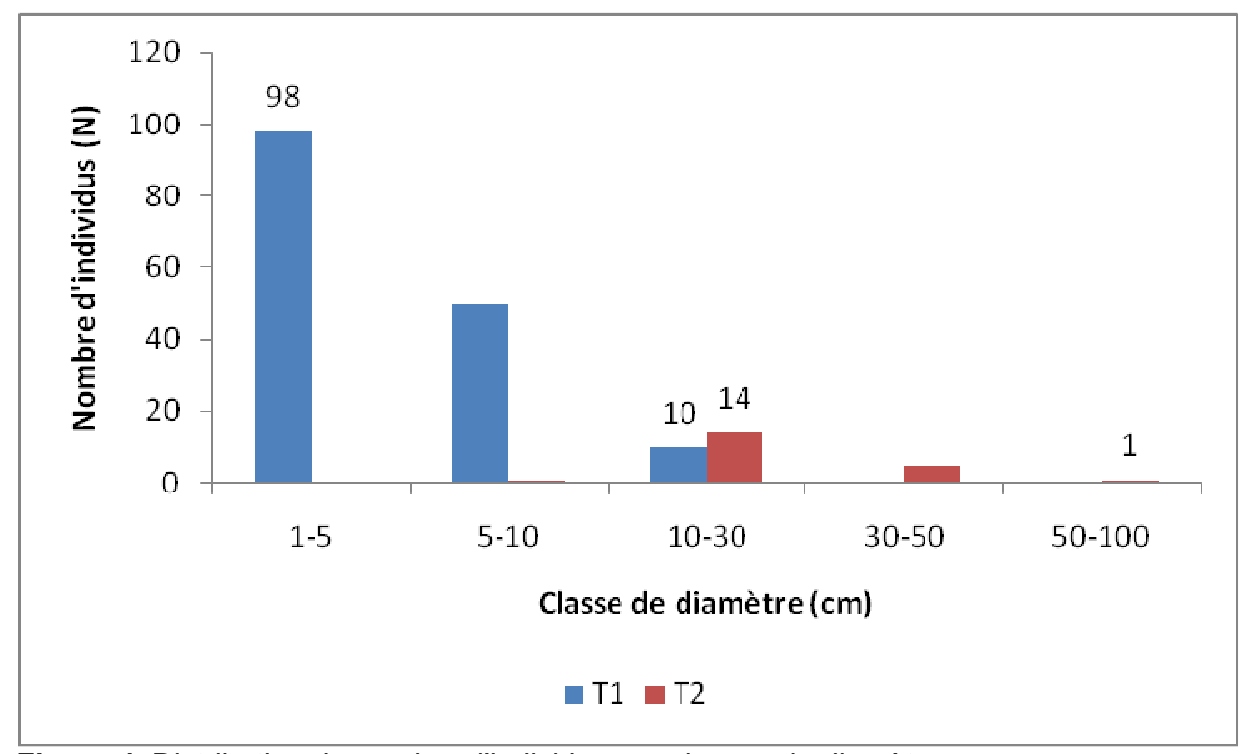

Figure 4: Distribution du nombre d'individus par classes de diamètre

Les hauteurs tout comme les diamètres se repartissent aussi en trois (T1) et quatre classes (T2). Les classes de hauteur modale sont respectivement 1-5 m ( N=102 soit $64,55 \%)$ et $10-30(\mathrm{~N}=16$ soit $76,19 \%)$ pour $\mathrm{T} 1$ et $\mathrm{T} 2$
. La classe de hauteur la plus grande pour le transect 1 est $10-30 \mathrm{~m}(\mathrm{~N}=4 ; 2,53 \%$ du nombre d'individus total $\mathrm{du}$ transect) contre $50-100 \mathrm{~m}(\mathrm{~N}=1 ; 4,76 \%)$ pour le transect2 ( $\mathrm{N}=21$ ) (Fig. 5). 


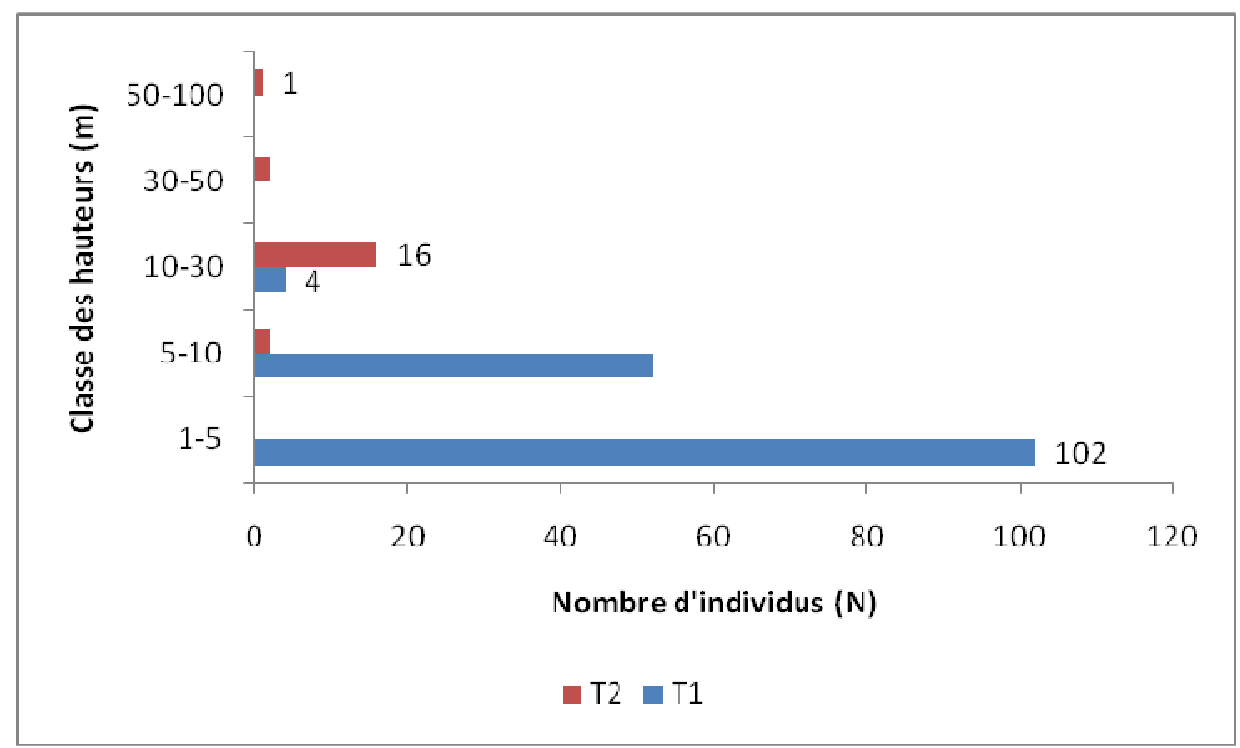

Figure 5: Distribution du nombre d'individus par classes de hauteur

Croissance du peuplement: La croissance annuelle des diamètres $(\mathrm{Cad})$ est de l'ordre de 0,13 $\pm 0,01 \mathrm{~cm} /$ an et $0,18 \pm 0,01 \mathrm{~cm} /$ an respectivement pour T1 et T2 pour la période 2009-2012. Elle est de $0,14 \pm 0,01 \mathrm{~cm} / \mathrm{an}$ et $0,29 \pm 0,05 \mathrm{~cm} / \mathrm{an}(2012-$ 2014) contre $0,14 \pm 0,01 \mathrm{~cm} / \mathrm{an}$ et $0,22 \pm$ 0,02cm/an (2009-2014). Les hauteurs montrent une croissance annuelle constante dans $\mathrm{T} 1$ ( $\mathrm{Cah}=$ $0,09 \mathrm{~m} / \mathrm{an})$ pendant les trois périodes alors qu'elle croit dans T2 (Cah $=0,13 \pm 0,01$ et $0,21 \pm 0,04 \mathrm{~m}$ ) pour les périodes 2009-2012 et 2012-2014. La croissance annuelle de la surface basale (Cas) montre une évolution croissance dans T1 $(0,017$ et $\left.0,08 \mathrm{~m}^{2} / \mathrm{ha} / \mathrm{an}\right)$ et T2 $\left(0,23\right.$ et $\left.0,26 \mathrm{~m}^{2} / \mathrm{ha} / \mathrm{an}\right)$ pendant les périodes consécutives 2009-2012 et 2012-2014. Elle est identique entre T1 et T2 (Cas $=0,017$ ) pour la période 2009-2014. La croissance annuelle du volume montre aussi une évolution croissante pour $\mathrm{T} 1(0,08$ et 0,09 $\left.\mathrm{m}^{3} / \mathrm{ha} / \mathrm{an}\right)$ et T2 $\left(0,26\right.$ et $\left.0,45 \mathrm{~m}^{3} / \mathrm{ha} / \mathrm{an}\right)$ pour les deux périodes consécutives (Tableau 2). Afin d'examiner le dégré de signification des valeurs de la croissance du peuplement, le one way anova test a été utilisé. II a révélé une différence non significative $(P>0,05)$ de la croissance annuelle dans les transect 1 et 2

Tableau 2 : Croissance annuelle par période du peuplement

\begin{tabular}{|c|c|c|c|c|c|c|c|}
\hline \multirow[b]{2}{*}{ Période } & \multirow[b]{2}{*}{ Transect } & \multicolumn{6}{|c|}{ Croissance annuelle } \\
\hline & & $\begin{array}{l}\text { Cad } \\
\text { (cm/an) }\end{array}$ & Écart type & Cah (m/an) & $\begin{array}{l}\text { Écart } \\
\text { type }\end{array}$ & Cas (m²/ha/an) & $\operatorname{Cav}\left(m^{3} / \mathrm{ha} / \mathrm{an}\right)$ \\
\hline & T1 & 0,13 & 0,01 & 0,09 & 0,008 & 0,017 & 0,08 \\
\hline $\begin{array}{l}2009- \\
2012\end{array}$ & T2 & 0,18 & 0,01 & 0,13 & 0,01 & 0,23 & 0,26 \\
\hline & $\mathrm{T} 1$ & 0,14 & 0,01 & 0,09 & 0,01 & 0,08 & 0,09 \\
\hline $\begin{array}{l}2012- \\
2014\end{array}$ & $\mathrm{~T} 2$ & 0,29 & 0,05 & 0,21 & 0,04 & 0,26 & 0,45 \\
\hline & T1 & 0,14 & 0,01 & 0,09 & 0,007 & 0,017 & 0,08 \\
\hline $\begin{array}{l}2009- \\
2014\end{array}$ & T2 & 0,22 & 0,02 & 0,16 & 0,01 & 0,017 & 0,34 \\
\hline
\end{tabular}


La distribution de la croissance annuelle des diamètres par classe de diamètre montre une évolution croissante pour les individus de petites classes $(1-5$ et $5-10 \mathrm{~cm})$. Entre la classe 5-10 et $30-50 \mathrm{~cm}$, cette évolution est décroissante dans $\mathrm{T} 1$ alors qu'elle est décroissance entre les classe 5-10 et 10-30 et constante entre les classes $10-30$ et $30-50 \mathrm{~cm}$ dans T2 (Fig. 6)

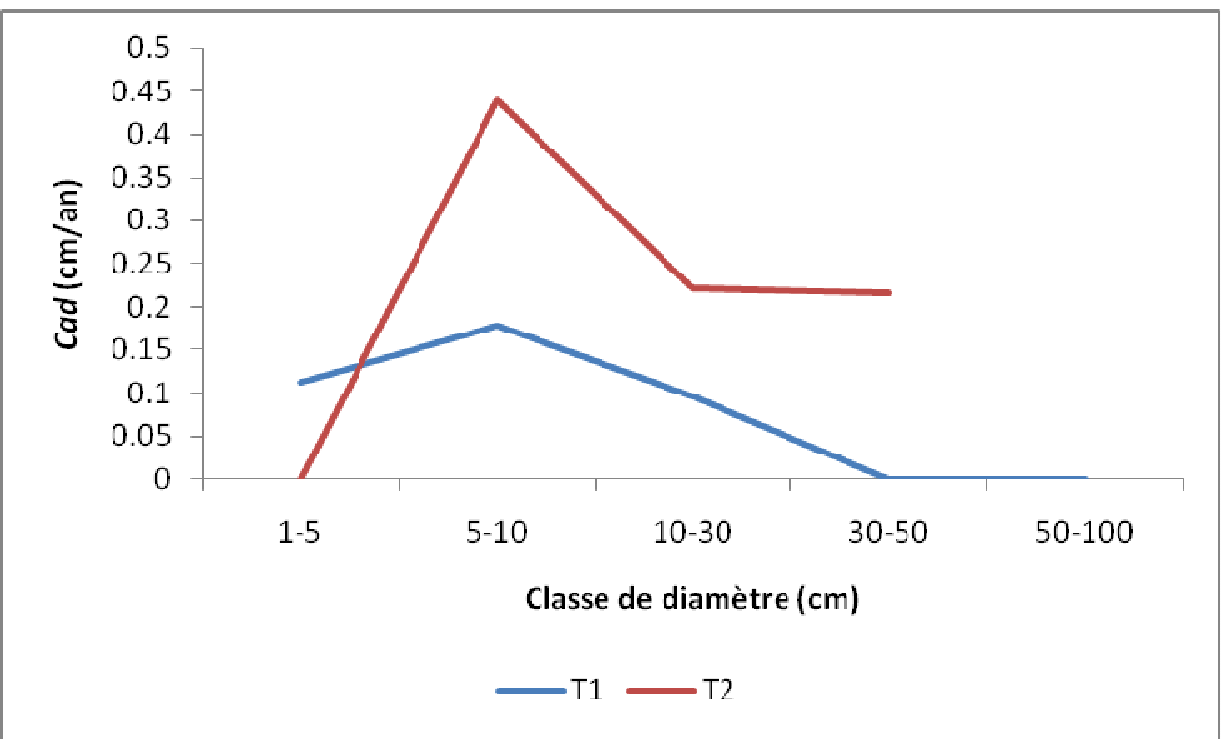

Figure 6: Distribution de la croissance annuelle des diamètres par classe

Cette évolution décroissance s'explique mieux par la mortalité naturelle et anthropique des individus que par le rétrécissement du diamètre des arbres. Ainsi les diamètres d'exploitation $d u$ bois de mangrove affectionnés par les riverains du site se situeraient dans l'intervalle $5-30 \mathrm{~cm}$ constitutive des classe $5-10$ et10$30 \mathrm{~cm}$. La stabilité de la croissance dans les grandes classes $(10-30$ et $30-50 \mathrm{~cm})$ dans T2 explique aussi bien la maturité des arbres que le caractère naturel (non perturbé) des mangroves de ce transect.
Mortalité : Le nombre d'individus mort ( $\mathrm{Nm})$ pour la période 2009-2012 était de 8 pour T1 et 1 pour T2. Pour la période 2012-2014, Nm est de 10 pour T1, aucun individu mort n'étant inventorié dans T2. De 2009 à 2014, le nombre d'arbre total mort est de 18 dans T1 et 01 dans T2. A l'hectare cette mortalité est de 133 pour T1 et 17 pour T2 pendant la période 20092012 contre 167 (T1) pour 2012-2014. La mortalité totale de 2009 à 2014 est de $300 \mathrm{Nm} /$ ha pour T1 contre $17 \mathrm{Nm} /$ ha pour T2 (Tableau 3).

Tableau 3 : Mortalité par période entre les différents transects

\begin{tabular}{lclll}
\hline \multirow{2}{*}{ Période } & & \multicolumn{3}{c}{ Mortalité } \\
\cline { 3 - 5 } $\mathbf{2 0 0 9 - 2 0 1 2}$ & Transect & $\mathrm{Nm}$ & $\mathrm{Nm} / \mathrm{ha}$ & $\mathrm{m}(\% / \mathrm{an})$ \\
& $\mathrm{T} 1$ & 8 & 133 & 1,68 \\
$\mathbf{2 0 1 2 - 2 0 1 4}$ & $\mathrm{T} 2$ & 1 & 17 & 1,58 \\
& $\mathrm{~T} 1$ & 10 & 167 & 3,33 \\
$\mathbf{2 0 0 9 - 2 0 1 4}$ & $\mathrm{T} 2$ & 0 & 0 & 0 \\
& $\mathrm{~T} 1$ & 18 & 300 & 2,27 \\
& $\mathrm{~T} 2$ & 1 & 17 & 0 \\
\hline
\end{tabular}

Le taux de mortalité annuelle $(\mathrm{m})$ de la période 2009 2012 est de 1,68\% (T1) et 1,58 (T2) contre 3,33\% (T1) et $0 \%$ (T2) pour la période 2012-2014. Le taux de mortalité moyenne annuelle de 2009 à 2014 est de 2,27\% (Tableau 3). 

mangroves de l'estuaire du Rio del Rey : Site de Bamusso (Sud-Ouest Cameroun)

\section{DISCUSSION}

L'étude sur la mangrove de l'estuaire du Rio del Rey a porté sur les paramètres de structure spatiale de la végétation (Fuhr, 1999). Les populations végétales étudiées ont montrés les dimensions variés avec des emplacements bien définis ce qui est en accords avec les travaux de Dajoz R (2000). En 2014 les diamètres moyens (Dmoy) sont de $5,43 \pm 0,28 \mathrm{~cm}$ (T1) et $25,26 \pm 1,93 \mathrm{~cm}$ (T2) alors que les hauteurs moyennes (Hmoy) sont de 4,98 $\pm 0,2 \mathrm{~m}$ (T1) et 19,29 $\pm 1,39 \mathrm{~m}$ (T2). L'étude des paramètres de structure du genre Rhizophora des mangroves de l'estuaire du Wouri (Din et al, 2008) a révélé les valeurs de $18 \mathrm{~cm}$ (Dmoy) et de $10 \mathrm{~m}$ (Hmoy). Ces résultats sont supérieurs à ceux du transect1 et inférieurs à ceux du transect2 de la présente étude. Un constat similaire est à établir entre les résultats de cet étude et ceux obtenus par Nfotabong (2011) qui a trouvés 20,92 cm (Dmoy) et $18,46 \mathrm{~m}$ (Hmoy) dans les mangroves de la zone périurbaine de Douala. Le diamètre moyen du transect1 $(4<$ Dmoy<10) montre que sa structure est constitués des individus moyens appartenant à la strate des microphanérophytes $(2<\mathrm{Hmoy}<10)$ qui est composé des arbustes. Le transect2 quand à lui appartient à la structure à gros individus(Dmoy>10) de la strate des mésophanérophytes $(10<\mathrm{Hmoy}<30)$ constitués des arbres (Lavigne et Magdelaine, 2005). D'après Ajonina (2008), le diamètre moyen du transect1 $(5<$ Dmoy $<10)$ place ses individus dans le

\section{CONCLUSION}

L'étude sur la dynamique de croissance et taux de mortalité de Rhizophora spp. dans les mangroves de l'estuaire du Rio del Rey montre que les individus d'une même espèce, situés à divers endroits, peuvent présenter les paramètres structuraux distincts les uns les autres. Un total de 179 individus appartenant tous au genre Rhizophora ont été répertorié en 2009 et l'analyse aussi bien de leur diamètre, hauteur ou densité absolue montre que le transect1 est constitué des individus jeunes alors que le transect2 comprend les individus plus matures. Aussi la surface basale du transect2 constitués des individus à gros diamètre varie très brusquement d'une campagne à l'autre en cas de disparition ou mortalité d'un de ces individus. Le taux de mortalité relativement faible présage aussi bien d'un

\section{REMERCIEMENTS}

Nous exprimons notre gratitude à I'ONG Cameroon Wildlife Conservation Society (CWCS) qui a facilité la groupe des perches alors que ceux du transect2 $(10<$ Dmoy<30) se retrouvent dans le groupe des poteaux. La relation entre le diamètre et la hauteur classe les individus du transect1 dans le facies mature $(5<$ Dmoy< $10 ; 2<$ Hmoy $<7)$ et les individus du transect2 dans le facies ancien (Lebigre, 1990; Ondo, 2006).

Les résultats de la densité absolue $(D a)$ expriment généralement la compétition moyenne dans le peuplement (Favrichon et al,1998). Ses valeurs sont de $2333 \mathrm{~N} / \mathrm{ha}$ pour T1 et $333 \mathrm{~N} / \mathrm{ha}$ pour T2 en 2014. Les mangroves de l'estuaire du Rio del Rey auraient donc des densités absolues supérieures à celles des mangroves de la zone périurbaine de Douala $(D a=95$ $\mathrm{N} / \mathrm{ha}$ ) pour le genre Rhizophora (Nfotabong, 2011). Par contre ces densités sont inférieures à celle des mangroves du Gabon ( $D a=15200 \mathrm{~N} / \mathrm{ha}$ ) pour le même genre (Ondo, 2006). La surface terrière ou surface basale $(S b)$ dans ce travail exprime la somme des surfaces sur un hectare des sections transversales des troncs des arbres effectués à $1,30 \mathrm{~m}$ du sol et son interprétation est fortement lié à la grosseur (diamètre) des individus (Jenner, 2002). Ses valeurs pour le transect1 $\left(S b=0,42 \mathrm{~m}^{2} / \mathrm{ha}\right)$ et pour le transect 2 $\left(S b=1,12 \mathrm{~m}^{2} / \mathrm{ha}\right.$ ) sont nettement plus petites que celles trouvées par Ondo en 2006 ( $S b=251,99 \mathrm{~m}^{2} / \mathrm{ha}$ ) et par Nfotabong en 2011 ( $S b=49,31 \mathrm{~m}^{2} / \mathrm{ha}$ ) pour le même genre Rhizophora.

bon taux de recrutement que d'une pression anthropique faible sur cet écosystème. Ce site d'étude s'avère être peu perturbé et il importe d'accentuer des mesures de conservation pour le pérenniser. Les études similaires sont à encourager dans les autres localités du Rio del Rey pour le même genre ainsi que les autres genres caractéristiques des mangroves. $\mathrm{Si}$ ces études arrivaient à démontrer le caractère peu perturbé de cet écosystème comme c'est le cas de la présente, les mangroves du Rio del Rey pourraient jouer un rôle prépondérant pour atténuer le phénomène de changement climatique aussi bien par le freinage de l'avancée de la mer que par la séquestration du carbone de ces différentes composantes.

logistique et les mesures des différents paramètres sur le terrain. Nous remercions aussi tous nos guides, 


\section{Ndema et al.. J. Appl. Biosci. Dynamique de croissance et taux de mortalité de Rhizophora spp. dans les}

mangroves de l'estuaire du Rio del Rey : Site de Bamusso (Sud-Ouest Cameroun)

pilotes d'embarcation et toute la population de Bamusso pour leurs assistance et hospitalité durant

\section{RÉFÉRENCES BIBLIOGRAPHIQUES}

Adame MF, Kauffman JB, Medina I, Gamboa JN,Torres O, 2013. Carbon stocks of tropical coastal wetlands within the Karstic Landscape of the Mexican Caribbean. PLOS ONE 8(2): e56569. doi:10.1371/journal.pone.0056569

Ajonina GN, 2008. Inventory and modelling mangrove forest stand dynamics following different levels of wood exploitation pressures in the DoualaEdea atlantic coast of Cameroon, Central Africa. PhD Thesis. Faculty of forest and environmental sciences, Albert-LudwigsUniversität Freiburg im Breisgau, Germany.232p.

Ajonina GN, Jin E, Mekongo F, Ayissi I, Usongo L, 2005. Gender roles and economics of exploitation, processing and marketing of bivalves and impacts on forest resources in the Douala-Edaa Wildlife Reserve, Cameroon. International Journal of Sustainable Development and World Ecology 12(2005): 161- 172.

Ajonina GN. and Usongo L, 2001. Preliminary Quantitative impact assessment of wood extraction on the mangroves of Douala-Edéa forest reserve, Cameroun. Tropical Biodiversity 7(2)3: 137-149.

Alongi DM, 2008. Mangrove forests: Resilience, protection from tsunamis, and responses to global climate change. Estuarine Coastal and Shelf Science 76: 1-13.

Cannicci S, Burrows D, Fratini S, Lee SY, Smith III TJ, Offenberg J, Dahdouh-Guebas F, 2008. Faunistic impact on vegetation structure and ecosystem function in mangrove forests. Aquat. Bot. 89(2): 186-200.

Dahdouh-Guebas F, 2006. Mangrove forests and tsunami protection. In : McGraw-Hill (éd). Yearbook of Science \& Technology, New York, USA. Pp: 187-191.

Dajoz R, 2000. Précis d'écologie. Paris: Dunod, 7e éd.,615p.

Din N, Puig H, Blasco F, 2006. Exploitation du bois dans les mangroves de Douala (Cameroun). Ann. Fac. Sci. Univ. Ydé I, série Sc. Nat. \& Vie 36(3): 89-103.

Dittmar T, Lra RJ, Kattner G, 2001. River or mangrove? Tracing major organic matter sources in l'étude. Nous n'oublierons pas tous les chercheurs qui ont contribué à rehausser la qualité de ce travail.

tropical Brazilian coastal waters. Marine Chemistry 73: 253-271.

Donato DC. and Kauffman JB, 2012. Protocols for the measurement, monitoring and reporting of structure, biomass and carbon stocks in mangrove forests. Working Paper 86. CIFOR, Bogor, Indonesia.

Duke NC, Meynecke JO, Dittmann S, Ellison AM, Anger K, Berger U, Cannicci S, Diele K, Ewel $\mathrm{KC}$, Field CD, Koedam N, Lee SY, Marchand C, Nordhaus I, Dahdouh-Guebas F, 2007. A world without mangroves? Science 317: 41-42.

Duke NC, 1992. Mangrove floristics and biogeography. In: Robertson, Al., Alongi, DM., (eds) Tropical mangrove ecosystems, Washington DC, American Geophysical Union, USA, pp. 63100.

Fuhr M, 1999. Structure et dynamique de la foret côtière du Gabon: Implication pour une succession secondaire dérivant de la foret monodominante à Okoumé (Aucoumea klaineana Pierre). Montpellier, Université des Sciences et Techniques du Languedoc Montpellier II, 183p.

Gattuso JP, Frankignoulle M, Wollast R, 1998. Carbon and carbonate metabolism in coastal aquatic ecosystems. Annual Rewiews 12: 405-433.

Hong PN. and San HT, 1993. Mangrove of Vietnam. IUCN, Gland Switzerland.

Imbert D, 1985. Organisation spatio-temporelle des communautés végétales dans la mangrove $\mathrm{du}$ grand Cul-de- sac marin (Guadeloupe). Université de Montpellier II, Thèse de doctorat, $132 p+$ annexes.

Jayatissa LP, Dahdouh-Guebas F, Koedam N, 2002. A review of the floral composition and distribution of mangroves in Sri Lanka. Botanical Journal of the Linnean Society 138: 29-43.

Jenner $X, 2002$. Décrire les peuplements feuillus issus du TSF. Forets de France, no 458, pp.35-37

Kovacs JM, 1999. Assessing mangrove use at the local scale. Landscape and Urban Planning 43: 207-208.

Krauss KW, Lovelock CE, McKee KL, Lopez-Hoffman L, Ewe SM, Sousa WP, 2008. Environmental drivers in mangrove establishment and early development. Aquat. Bot. 89(2): 105-127. 
Laegdsgaard P. and Johnson C, 2001. Why do juvenile fish utilise mangrove habitats? Journal of Experimental Marine Biology and Ecology 257: 229-253.

Lavigne F. and Magdelaine $\mathrm{CH}$, 2005. Typologie et organisation structurale des formations végétales. $\quad \mathrm{http}: / / \mathrm{www}$.notre planete.info/geographe/org plantes_o.php,3p.

Lebigre JM, 1990. Les marais maritimes du Gabon et de Madagascar. Contribution à une étude d'un milieu naturel tropical.Univ.de Bordeaux III, Thèse de doctorat d'État, 3 tomes, 615P.

Lovelock CE, Ruess RW, Feller IC, 2011. $\mathrm{CO}_{2}$ Efflux from Cleared Mangrove Peat. PLoS ONE 6(6): e21279. doi:10.1371/journal.pone.0021279

Nagelkerken I, Blaber S, Bouillon S, Green P, Haywood M, Kirton LG, Meynecke JO, Pawlik J, Penrose HM, Sasekumar A, Somerfield PJ, 2008. The habitat function of mangroves for terrestrial and marina fauna. Aquat. Bot. 89(2): 201-219.

Nfotabong $A A, 2011$. Impacts des activités anthropiques sur la structure de la végétation des mangroves de kribi, de l'embouchure du fleuve nyong et de l'estuaire du Cameroun. Thède de doctorat $\mathrm{PhD}$. Faculté des Sciences, Département de biologie des organismes, Laboratoire d'écologie

des systèmes et gestion des ressource. 255p.
Ondo A E, 2006. Dynamique des paysages végétaux du littoral centre-ouest du Gabon autour de Port-Gentil : Approche spatiale et analyse des données de terrain. Thèse de doctorat. Univ. Paul-Valery Montpellier 3, France. $302 \mathrm{p}$.

Spalding MD, Blasco F, Field CD, 1997. World Mangrove Atlas. ISME, Okinawa, Japan. 251 p.

Thevand A, 2002. Structure et dynamique des mangroves de la région de Kaw (Guyane Française), Étude par télédétection et analyse in situ. Mémoire de DEA, UPS-Toulouse III, France. $31 \mathrm{p}$.

Tomlinson PB, 1986. The botany of mangroves. Cambridge University Press, $413 \mathrm{p}$.

Trevor GJ, Harifidy RR, Lalao R, Garth C, Adia B, 2014. Ecological Variability and Carbon Stock Estimates of Mangrove Ecosystems in Northwestern Madagascar. Forests 2014, 5, 177-205; doi:10.3390/55010177

Twilley RR, Chen RH, Hargis T, 1992. Carbon sinks in mangroves and their implications to carbon budget of tropical coastal ecosystems. Water, Air and Soil Pollution 64: 265-288.

Walters BB, Rönnbäck P, Kovacs J, Crona B, Hussain $\mathrm{S}$, Badola R, Primavera JH, Barbier EB, Dahdouh-Guebas F, 2008. Ethnobiology, socio-economics and adaptive management of mangroves. Aquat. Bot. 89(2): 220-236. 